\title{
Research on the Pollution of Express Plastic Packaging Based on System Dynamics
}

\author{
Guoqiang Ren ${ }^{1}$, Cuiming Zhao ${ }^{1, *}$ \\ ${ }^{1}$ School of Management, Tianjin University of Technology, 300384Tianjin, China
}

\begin{abstract}
With the rapid development of e-commerce, the environmental pollution caused by express plastic packaging is increasingly prominent. This paper takes express plastic packaging as the research object and analyzes its harm to the environment. Under the guidance of green logistics, using the research method of system dynamics, the causal loop diagram of express plastic packaging system is drawn and analyzed. Finally, based on the circuit diagram, the paper puts forward some measures, such as strengthening the construction of laws and regulations, improving the recycling system, strengthening research and development, industry standardization and focusing on publicity, to reduce the environmental pollution caused by express packaging.
\end{abstract}

\section{Introduction}

The rapid development of e-commerce has brought a lot of employment opportunities and economic income, but also brought serious plastic pollution. In 2018, China's express industry consumed 24.5 billion plastic bags, and a large number of express packages brought great challenges to the control of plastic pollution. Therefore, we should take the concept of green logistics as a guide, from the perspective of environmental protection, to achieve the unity of economic interests, social interests and environmental interests of the logistics industry.

Lei Chen and Hongli Mu (2019) thought that the plastic packaging bags used by most express companies had little recyclability and could only be sent to landfill for burial, which would have adverse effects on the environment and people's health ${ }^{[1]}$. Feiyu Chen \& Hong Chen et al (2018), based on individual behavioral psychological mechanism, concluded that individuals with high psychological perception and strong awareness of prevention are more likely to participate in express package garbage collection [2]. Yanping Wang and Yixiang Deng et al (2019) proposed to manage the whole life cycle of disposable plastics from production, use to recycling ${ }^{[3]}$.

The research of scholars provides a great reference value for this paper. This paper uses the method of system dynamics to construct the causal loop diagram of express plastic packaging system, and proposes some measures based on the loop diagram in order to reduce the pollution of express packaging.

\section{Some situations of express plastic packaging}

\subsection{Express business volume}

As shown in the following chart, in just five years from 2014 to 2018, the volume of express business in China has grown rapidly, from 13.96 billion to 50.71 billion, which has nearly doubled. It is estimated that the volume of express business in China will exceed 60 billion in 2019 . With the massive express delivery, not only the huge revenue, but also the environmental pollution caused by the express packaging.

Table 1. 2014-2019 Chinese express business volume (unit: 100 million pieces)

\begin{tabular}{|c|c|c|c|c|c|c|}
\hline Year & 2014 & 2015 & 2016 & 2017 & 2018 & $2019 \mathrm{E}$ \\
\hline Volume & 139.6 & 206.7 & 312.8 & 400.6 & 507.1 & 600 \\
\hline
\end{tabular}

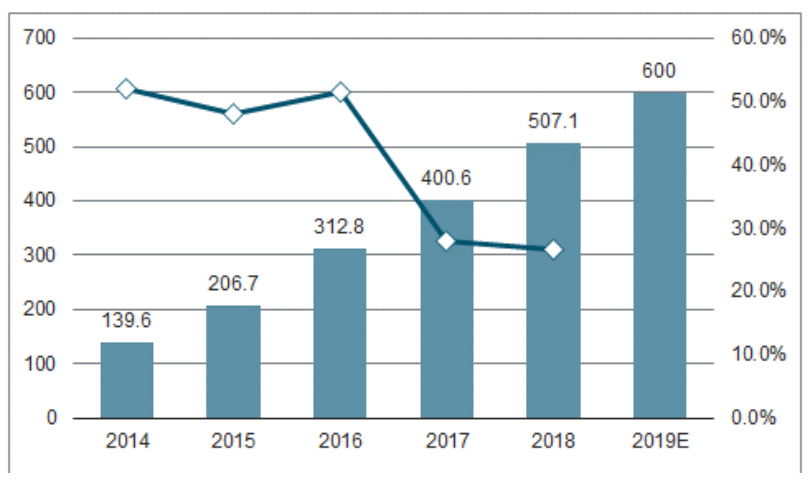

Fig. 1. 2014-2019 Chinese express business volume (unit: 100 million pieces)

\footnotetext{
* Corresponding author: xmsm_cuiming@163.com
} 


\subsection{Brief introduction of express plastic packaging}

Plastic products have the advantages of small weight, high plasticity, waterproof, mature production technology, and low cost. They are the first choice of packaging materials for Internet sellers and express companies. At present, the main components of express packaging plastics are $\mathrm{PE}$ (polyethylene) and LDPE (low-density polyethylene), which can be roughly divided into recycled materials and new materials according to the type of use ${ }^{[4]}$. These plastic packages are different in color and material. Black bags are made of recycled materials, and the price is the cheapest. Green is half recycled and half brand new. White is brand new material, and the price is more than twice that of black grey. In order to save costs, most of the packaging bags used by express companies are inferior black gray plastic bags made of chemical materials, domestic garbage and medical garbage ${ }^{[5]}$.

In order to improve the performance of express plastic packaging, at present many enterprises add a lot of toxic and harmful substances such as flame retardant and plasticizer into the packaging materials, and even use poor quality ink for printing. Through contact with human body, these express plastic packages will cause great harm to human health ${ }^{[6]}$. Most of the plastic packages of express delivery will be thrown into the garbage can and sent to the landfill together with other garbage. The degradation time of plastic waste is up to $400-500$ years, which is far longer than the design service life of most landfills for 200 years. If these express packages are exposed in the environment, they will cause pollution to the environment. If the plastic discarded in the environment is decomposed into micro plastic particles, it can enter the organism with the behavior of food consumption, and finally affect the health of human beings through the food chain ${ }^{[7]}$.

\section{System dynamics model}

\subsection{Model building}

There are many variables that affect the pollution of express plastic packaging. Feiyu Chen \& Hong Chen et al (2018) and others take psychological authorization as variables ${ }^{[8]}$, Fangwei Liu and Ning Ba et al (2019) analyzed the use of biodegradable materials in express packaging ${ }^{[9]}$, Yanping Wang and Yixiang Deng et al (2019) summarized the policies and laws issued by countries in the world ${ }^{[10]}$. In addition, pollution control input, standardization and recycling containers and other factors will also affect the packaging pollution of express delivery. According to the above variables, Vensim is used to establish a causal loop diagram of express plastic packaging system. This paper analyzes five loops.

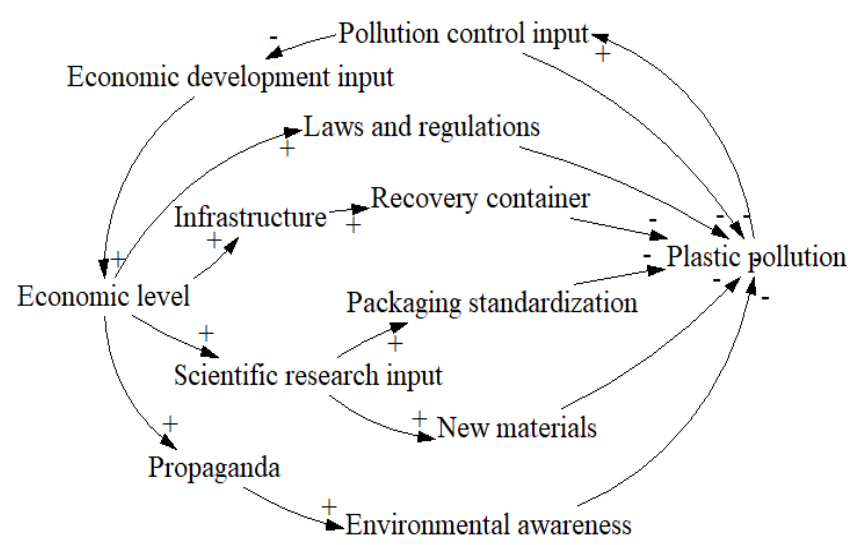

Fig. 2. Cause and effect circuit diagram of express plastic packaging system

\subsection{Model analysis}

(1) Economic level $\rightarrow$ Laws and regulations $\rightarrow$ Plastic pollution $\rightarrow$ Pollution control input $\rightarrow$ Economic development input $\rightarrow$ Economic level.

This is a positive feedback loop. The economic foundation determines the superstructure. With the improvement of the economic development level, the continuous development of political civilization, and the continuous improvement of the laws related to pollution, the pollution caused by the plastic package of express delivery will gradually become smaller. If more and more express plastic packaging pollutes the environment, the government will spend a lot of money to control the pollution, which will lead to the reduction of funds for economic development, and then make the level of economic development decline.

(2) Economic level $\rightarrow$ Infrastructure $\rightarrow$ Recovery container $\rightarrow$ Plastic pollution $\rightarrow$ Pollution control input $\rightarrow$ Economic development input $\rightarrow$ Economic level.

This loop is a positive feedback loop. With the improvement of the economic level, the infrastructure construction is more and more perfect, more express packaging recycling containers are arranged, some express packaging can be reused, thus reducing the environmental pollution of express packaging.

(3) Economic level $\rightarrow$ Scientific research input $\rightarrow$ Packaging standardization $\rightarrow$ Plastic pollution $\rightarrow$ Pollution control input $\rightarrow$ Economic development input $\rightarrow$ Economic level.

This is a positive feedback loop. With the improvement of economic development level, the funds for scientific research are increasing gradually. Researchers can design standardized express packaging plastics. These standardized packaging plastics have good versatility and high utilization rate, which can reduce the pollution of plastics to the environment.

(4) Economic level $\rightarrow$ Scientific research input $\rightarrow$ New materials $\rightarrow$ Plastic pollution $\rightarrow$ Pollution control input $\rightarrow$ Economic development input $\rightarrow$ Economic level.

This loop is a positive feedback loop. The improvement of economic development level drives the increase of scientific research investment, and researchers will develop new packaging materials. If the new packaging material has good degradability, little 
environmental pollution and good performance, it can replace the plastic packaging material and reduce the harm of express plastic packaging to the environment.

(5) Economic level $\rightarrow$ Propaganda $\rightarrow$ Environmental awareness $\rightarrow$ Plastic pollution $\rightarrow$ Pollution control input $\rightarrow$ Economic development input $\rightarrow$ Economic level.

This is a positive feedback loop. With the improvement of economic level, more and more publicity has been given to express plastic packaging, and people's awareness of environmental protection has been gradually enhanced. People actively respond to the call of the government to minimize the environmental damage caused by express plastic packaging.

\section{Conclusions and Suggestions}

\subsection{Conclusions}

This paper first introduces the situation and harm of express plastic packaging, then constructs the cause and effect loop diagram of express plastic packaging system, and analyzes the loop in the system. The analysis found that improving regulations and recycling systems, studying new environmental materials and industry standards, and raising public awareness can reduce environmental pollution from packaging. The reduction of environmental pollution can promote the development of economy.

Finally, based on the above research, some suggestions for reducing packaging pollution are given.

\subsection{Suggestions}

Firstly, promote the construction of laws and regulations. The state should constantly improve the construction of laws and regulations related to express plastic packaging, and use the legal system to supervise the standardized operation of plastic packaging from use to recycling. In addition, we should strengthen the supervision of the implementation effect of the law and urge the express companies to follow the relevant laws. All departments of the government should also clarify their responsibilities and avoid prevarication. For enterprises, if they are actively implemented, they can be given preferential measures such as tax relief or financial subsidies, while if they are passively implemented, they will be punished.

Secondly, improve the express package recycling system. Implementation plan for promoting green packaging in express industry proposes to steadily promote the saving, full recycling and effective reuse of express industry packaging. The government can place the recycling containers of express packages in the residential communities, so that the residents can conveniently throw the plastic packages into the recycling containers, and then the express companies can uniformly recycle and distribute them. In addition, the system of simultaneous delivery and collection can also be implemented, that is, the courier will directly recycle the packaging materials from the customers after delivering the express ${ }^{[11]}$. Through the recovery of express package, it can not only increase the times of using express package, but also reduce the cost of express company.

Thirdly, increase the research of environmental protection materials. Packings for Express Service released in 2018 proposed that biodegradable plastic should be used for express packaging bags. Fully biodegradable plastics are mainly made of natural polymers such as starch and cellulose, which alleviate the dependence of plastics industry on petroleum. However, biodegradable plastics also face the disadvantages of high cost, poor performance and insufficient capacity. Therefore, we should strengthen the research of biodegradable plastics, improve the equipment and upgrade process, and constantly overcome its shortcomings, so that it can be widely used.

Fourthly, set industry standards. The express industry should establish a unified plastic packaging standard to lay a foundation for the recycling of express plastics. Only when the plastic package of express delivery is standardized can the plastic package be reused efficiently. For example, clothing packaging size and footwear packaging size form a uniform standard in the industry, so that the recycling of packaging bags can be directly applied to the corresponding goods.

Finally, strengthen propaganda. It is necessary for the public to take part in the control of pollution caused by express plastic packaging. The state should publicize the pollution caused by the plastic package of express delivery through TV, Internet and display board, so as to improve the public's awareness of the harm of plastic pollution. In addition, we should create a social atmosphere to protect the environment, enhance the residents' awareness of environmental protection, so that citizens can actively practice the social responsibility of reducing plastic pollution.

\section{Reference}

1. L. Chen, H. Mu, Environmental problems caused by express plastic packaging and Countermeasures. ETU, 32, 78-79(2019). (in Chinese)

2. F. Chen, H. Chen, J. Yang, et al, Impact of regulatory focus on express packaging waste recycling behavior: moderating role of psychological empowerment perception. ESPR, 26, 8862-8874(2019).

3. Y. Wang, Y. Deng, C. Zhang, et al, Study on the Management Policies of Single-Use Plastics Pollution in China. RES. (to be published)

4. J. Wang, T. Cao, Talk about the present situation of express package and its solution. LIST, 32, 129130(2016). (in Chinese)

5. A. $\mathrm{Xu}, \mathrm{A}$ Probe into the Packaging Problems of Express Mail in Online Shopping. LEAM, 37, 126128(2015).

6. Y. Gong, H. Li, Study on environmental cost of express package under the background of ecological civilization construction. YXJ, 47, 127-128(2019). (in Chinese)

7. Y. Deng, K. Lei, L. An, et al, Countermeasurces on Control of Plastic Litter and Microplastic Pollution. 
Bull Chin Acad Sci, 33, 1042-1051(2018).

8. F. Liu, N. Ba, Z. Zhen, et al, Introduction of Biodegradable Materials for Express Packaging. PP, 29, 16-18+15(2019).

9. H. Sun, On the recovery and reuse of express package. XDYX, 80-81(2018). 